\title{
STOCHASTIC CHAOTIC MOTION OF SHIPS IN BEAM SEAS
}

Li-Qin Liu

Department of Naval Architecture \& Ocean Engineering, Tianjin University, Tianjin 300072, P.R.C., liu_liqin@eyou.com

You-Gang Tang

Department of Naval Architecture \& Ocean Engineering, Tianjin University, Tianjin 300072, P.R.C.

Hong-Xia Li

Department of Naval Architecture \& Ocean Engineering, Tianjin University, Tianjin 300072, P.R.C.

Follow this and additional works at: https://jmstt.ntou.edu.tw/journal

Part of the Mechanical Engineering Commons

\section{Recommended Citation}

Liu, Li-Qin; Tang, You-Gang; and Li, Hong-Xia (2007) "STOCHASTIC CHAOTIC MOTION OF SHIPS IN BEAM SEAS," Journal of Marine Science and Technology. Vol. 15: Iss. 2, Article 7.

DOI: $10.51400 / 2709-6998.2040$

Available at: https://jmstt.ntou.edu.tw/journal/vol15/iss2/7

This Research Article is brought to you for free and open access by Journal of Marine Science and Technology. It has been accepted for inclusion in Journal of Marine Science and Technology by an authorized editor of Journal of Marine Science and Technology. 


\section{STOCHASTIC CHAOTIC MOTION OF SHIPS IN BEAM SEAS}

Acknowledgements

The authors are grateful to the National Natural Science Foundation of China for supporting this work (Grant No. 50279026). 


\title{
STOCHASTIC CHAOTIC MOTION OF SHIPS IN BEAM SEAS
}

\author{
Li-Qin Liu*, You-Gang Tang*, and Hong-Xia Li*
}

Key words: random chaotic motion, random melnikov mean-square criterion, path integral, probability density function.

\begin{abstract}
The stochastic chaotic motion and the threshold intensity of the external excitation force for onset of chaos of the ships in random beam waves are studied by the nonlinear stochastic dynamics theory. The random differential equation of ships' rolling motion is established with considering the nonlinear damping, nonlinear restoring moment and the white noise wave excitation. The random Melnikov mean-square criterion is used to determine the threshold intensity for onset of chaos. The probability density function of the rolling response is calculated through solving the stochastic differential equations by applying the path integral method in the chaotic region. It is found that the ships undergo stochastic chaotic motion when the real intensity of the white noise exceeds the threshold intensity, the stable probability density function of the roll response has two peaks and the random jump happens in the response of the system for high intensity of the white noise excitation. The chaotic response is further investigated via numerical results of the system.
\end{abstract}

\section{INTRODUCTION}

Ships will undergo chaotic motion before capsizing. Identifying the chaotic motion and the critical condition of it are very important for both predicting ships' capsizing and studying the capsize mechanism. People have done much work about the ships dynamics in regular waves and found the nonlinear phenomena of amplitude jumping, superharmonic and subharmonic response, period and chaotic motion of the ships $[8,9]$. There are some initial researches on the dynamic characteristics of ships in random waves, for example, Yim and Huan [11] analyzed the roll chaotic motion of ships under periodic excitation perturbed by random noise via the probability density function, Francescutto [2] studied the bifurcation and chaos of ships' rolling motion in narrow banded waves via the perturbation method of multiple time scale method and Hsieh and Troesch [3]

Paper Submitted 01/09/06, Accepted 04/30/06. Author for Correspondence: Li-Qin Liu.E-mail: liu_liqin@eyou.com.

*Department of Naval Architecture \& Ocean Engineering, Tianjin University, Tianjin 300072, P.R.C. studied the ships' chaotic motion by Melnikov function. There is no standard method that can be used to identify the ships chaotic motion in random waves.

The rolling chaotic motion of ships subjected to random beam waves is studied by the probability density function and the numerical method. The random Melnikov mean-square criterion is used to determine the threshold intensity of the external excitation force for onset of chaos. The joint probability density function of the roll response is calculated by solving the differential equation via the path integral method in the chaotic region. The chaotic characteristic is investigated via the shape of the probability density function and numerical results of the system when the real intensity of the white noise exceeds the threshold intensity.

\section{The stochastic nonlinear roll model of ships}

The governing equation can be expressed in the following form for ships in the beam wave

$$
\begin{aligned}
\left(I_{44}+\right. & \left.A_{44}(\omega)\right) \ddot{\varphi}+B_{44}(\omega) \dot{\varphi}+B_{44 q}(\omega) \dot{\varphi}^{3} \\
& +\Delta G Z_{m}(\varphi)=F_{\text {sea }}(\tau)
\end{aligned}
$$

Where $I_{44}$ is the moment of inertia of the ships about the roll axis, $A_{44}$ is the linear roll hydrodynamic added mass coefficient, $B_{44}$ is the linear roll damping coefficient, $B_{44 q}$ is the cubic damping coefficient, $F_{\text {sea }}(\tau)$ is the wave excitation force, $\Delta$ is the displacement, $G Z_{m}(\varphi)$ including the static effects of water-on-deck is a polynomial approximation to the designed roll righting arm curve $G Z(\varphi)$. For a special ship the details of how the modified roll restoring arm curve $G Z_{m}(\varphi)$ is determined are contained in the work of Falzarano [1].

The realistic wave is colored noise, for a special condition the wave excitation is treated as white noise in order to simplify the computation. The motion of ships with water-on-deck considered is divided into two dynamical regions-homoclinic and heteroclinic and the modified roll righting arm curve is approximated as $G Z_{m}(\varphi)=-C_{1} \varphi+C_{3} \varphi^{3}$ in the homoclinic region [1], Eq. (1) can be rewritten in the following non-dimensional 
form

$\ddot{\varphi}(t)+d_{1}^{\prime} \dot{\varphi}(t)+d_{3} \dot{\varphi}^{3}(t)-\varphi(t)+k_{3} \varphi(t)^{3}=\xi(t)$

Where $t=\omega_{0} \tau ; \omega_{0}=\sqrt{\frac{\Delta C_{1}}{I_{44}+A_{44}(\omega)}} ; d_{1}^{\prime}=\frac{B_{44}}{\Delta C_{1}} \omega_{0}$ and $d_{3}=\frac{B_{44 q}}{I_{44}+A_{44}(\omega)} \omega_{0}$ denote the linear and nonlinear damping coefficient respectively; $k_{3}=\frac{C_{3}}{C_{1}}$ is the nonlinear restoring moment coefficient; $\xi(t)=\frac{F_{\text {sea }}}{\Delta C_{1}}=\sqrt{D} N(t)$ denotes the white noise with intensity of $D ; N(t)=d W(t) /$ $d t$, where $W(t)$ is standard Wiener process. By performing equivalent linearization on the nonlinear damping force and replacing roll angle $\varphi$ and roll angular velocity $\dot{\varphi}$ with $x(t)$ and $y(t)$ respectively, Eq. (2) can be rewritten as

$$
\left\{\begin{array}{l}
\dot{x}(t)=y(t) \\
\dot{y}(t)=x(t)-k_{3} x(t)^{3}-d_{1} y(t)+\xi(t)
\end{array}\right.
$$

Here $d_{1}$ is the equivalent linearization damping coefficient. Eq. (3) can be rewritten as

$$
\left\{\begin{array}{l}
d x=y d t \\
d y=\left(-d_{1} y+x-k_{3} x^{3}\right) d t+\sqrt{D} d W(t)
\end{array}\right.
$$

The Wong-Zakai correction terms [12] of Eq. (4) is equal to zero and Eq. (4) can be approximated by $I t \hat{o}$ differential Eqs. as following

$$
\left\{\begin{array}{l}
d x=a_{1}(x, y, t) d t+b_{1}(x, y, t) d W(t) \\
d y=a_{2}(x, y, t) d t+b_{2}(x, y, t) d W(t)
\end{array}\right.
$$

Where, $\left\{\begin{array}{l}a_{1}=y, b_{1}=0 \\ a_{2}=\left(-d_{1} y+x-k_{3} x^{3}\right), b_{2}=\sqrt{D}\end{array}\right.$.

\section{The random melnikov mean-square criteria}

If the Melnikov function has a simple zero, it is known that the stable and unstable manifolds of the Poincare map have at least one transversal intersection and the system is chaotic in the Smale meaning [12]. As for the stochastic process, the Melnikov process should be studied in the probability perspective that is the random Melnikov mean-square criteria [5, 12]. Considering a single degree of freedom Hamiltonian system subjected to noise excitation

$$
\left\{\begin{array}{l}
\dot{Q}=\frac{\partial H}{\partial P} \\
\dot{P}=-\frac{\partial H}{\partial Q}-\varepsilon c(Q, P) \frac{\partial H}{\partial P}+\varepsilon f(Q, P) \zeta(t)
\end{array}\right.
$$

Where $H=p^{2} / 2+U(q), \zeta(t)$ is the white noise external excitation with zero mean, $c(Q, P)$ denotes the coefficient of damping, $\varepsilon$ is a small positive parameter. It is assumed that the Hamilton system associated with Eq. (6) possesses a hyperbolic fixed point connected by homoclinic orbits $\left(q_{0}(t), p_{0}(t)\right.$. The random Melnikov process for system (6) is

$$
\begin{aligned}
M\left(t_{0}\right) & =\int_{-\infty}^{+\infty} \frac{\partial H}{\partial P}\left[-c(Q, P) \frac{\partial H}{\partial P}+f(Q, P) \zeta\left(t+t_{0}\right)\right] d t \\
& =M_{d}+Z\left(t_{0}\right)
\end{aligned}
$$

Where $M_{d}=\int_{-\infty}^{+\infty}\left[-c(Q, P)\left(\frac{\partial H}{\partial P}\right)^{2}\right] d t, Z\left(t_{0}\right)$

$$
=\int_{-\infty}^{+\infty} \frac{\partial H}{\partial P}\left[f(Q, P) \zeta\left(t+t_{0}\right)\right] d t,
$$

$Q=q_{0}(t)$ and $P=p_{0}(t)$. The mean value of random Melnikov process (7) is

$$
\begin{aligned}
E\left[M\left(t_{0}\right)\right]= & E\left[\int_{-\infty}^{+\infty}-c(Q, P)\left(\frac{\partial H}{\partial P}\right)^{2}\right] d t \\
& +\int_{-\infty}^{+\infty} \frac{\partial H}{\partial P} \cdot f(Q, p) \cdot E\left[\zeta\left(t+t_{0}\right)\right] d t \\
= & -\int_{-\infty}^{+\infty} c(Q, P)\left(\frac{\partial H}{\partial P}\right)^{2} d t
\end{aligned}
$$

For $c(Q, p)>0$, Eq. (8) is negative, which indicates the system (6) can not be chaotic in the mean sense. The mean-square value of the random Melnikov process (7) is

$$
E\left[M^{2}\left(t_{0}\right)\right]=-\sigma_{M_{d}}^{2}+\sigma_{z}^{2}
$$

Where

$$
\sigma_{M_{d}}^{2}=\left(\int_{-\infty}^{+\infty} c(Q, P)\left(\frac{\partial H}{\partial P}\right)^{2} d t\right)^{2}
$$




$$
\begin{aligned}
\sigma_{z}^{2} & =E\left[Z^{2}\left(t_{0}\right)\right] \\
& =E\left[\left(\int_{-\infty}^{+\infty} f(Q, P)\left(\frac{\partial H}{\partial P}\right) \zeta\left(t+t_{0}\right) d t\right)^{2}\right]
\end{aligned}
$$

In Eq. (11), the integral $\int_{-\infty}^{+\infty} f(Q, P)\left(\frac{\partial H}{\partial P}\right) \zeta\left(t+t_{0}\right) d t=$ $h(t) * \zeta(t)$ is convolution integral and $h(t)=f(Q, P)$ $\left.\left(\frac{\partial H}{\partial P}\right)\right|_{Q=q_{0}(t), P=p_{0}(t)}$ can be regarded as the impulse response function of a time-variant linear system. Thus, we have

$$
\sigma_{z}^{2}=\int_{-\infty}^{+\infty}|H(\omega)|^{2} S_{\zeta}(\omega) d \omega
$$

Where $H(\omega)$ is the frequency response function, which is the Fourier transformation of the impulse response function $h(t), S_{\zeta}(\omega)$ is the spectral density function of $\zeta(t)$. The condition for the random process (7) to have a simple zero in the mean-square sense is

$$
\sigma_{z}^{2}=\sigma_{M_{d}}^{2}
$$

Eq. (13) is the criterion of judging the threshold condition for onset of chaos of system (6).

For the ships' large roll in the random waves, the joint response process $(\phi, \dot{\phi})$ can be looked as two degree of freedom Markov process, the probability transition process of the roll response can be computed via the path integral method $[6,7,10]$.

\section{Numerical examples}

Taking a barge with bilge keel designed as an example, the dimensionless parameters of it [4] are: $d_{1}$ $=0.185$ and $k_{3}=1$. Substituting the ship's parameters into Eq. (4), the threshold intensity for onset of chaos can be calculated by the stochastic Melnikov criteria (13) and the numerical result is obtained in Figure 1.

Figure 1 shows the threshold intensity of white noise for onset of chaos with the different $k_{3}$ and $d_{1}$. Simple zero of Melnikov function is only the necessary condition for onset of chaos. The chaotic characteristic of the ship is studied by the probability density function and roll response of the system.

According to Figure 1, the threshold intensity of the white noise for the barge's chaotic motion is $D=$ 0.0045 . The barge's probability density function for $D$ $=0.087$ is calculated. Assuming the initial probability density function obeys the Gaussian distributing, Itô stochastic differential Eq. (5) is solved by the path integral method within the domain of $x \in[-1.8,1.8]$ and $y \in[-1.8,1.8]$. The evolution of three-dimensional figures for the joint probability density function and the corresponding contour plots in the time domain are obtained in Figure 2 and Figure 3.

Figure 2 and Figure 3 show the evolution of the joint probability density function and the corresponding contour plots in the time domain, where $\operatorname{Jpdf}(\mathrm{x}, \mathrm{y})$ denotes the joint probability density function. The value of probability density function for roll response of the barge in each state can be obtained from Figure 2, therefore the probability that the barge system stays in the desired phase space region can be calculated conveniently. It is found that the initial probability density function has a single peak and one attraction region, here the attraction region is the state space around the peak whose probability density function is more than zero, after 15.15 seconds the maximal value of the probability density function holds 0.35 and the shape of it is invariable. So, the stable probability density function of the system is obtained and it has two connected peaks. That means the system has two high probability states and the response may jump from one state to another. The responses of the system are obtained in Figure 4 and Figure 5.

The roll response is shown in Figure 4 and Figure 5. It is found that the response of the system is restricted in one attraction region for the low intensity of white noise and the system undergoes stable chaotic motion, while it jumps randomly from one high probability state to another for the high intensity of white noise. That will lead ships to instability and even to capsizing.

\section{CONCLUSIONS}

The stochastic roll chaotic motion of the ships subjected to white noise waves is analyzed. The random Melnikov mean-square criterion is used to identify the

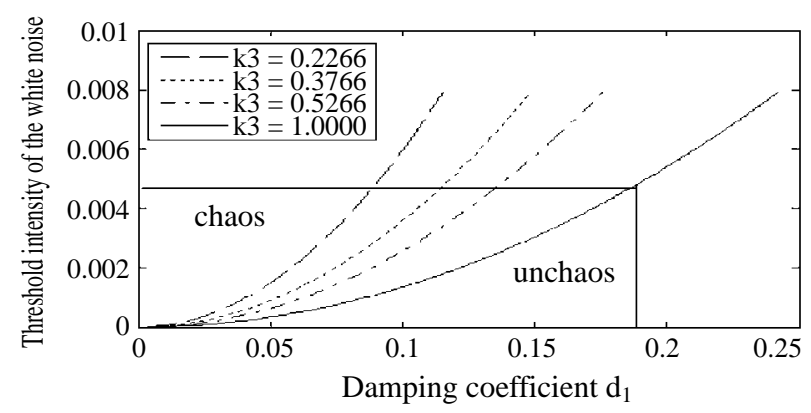

Fig. 1. The threshold intensity of white noise for onset of chaos. 


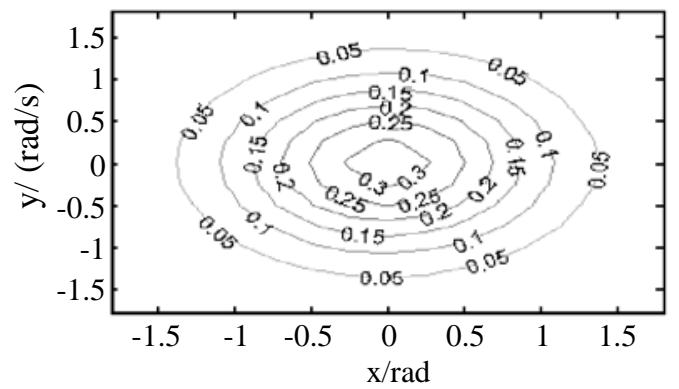

(a) $\mathrm{t}=0 / \mathrm{s}$

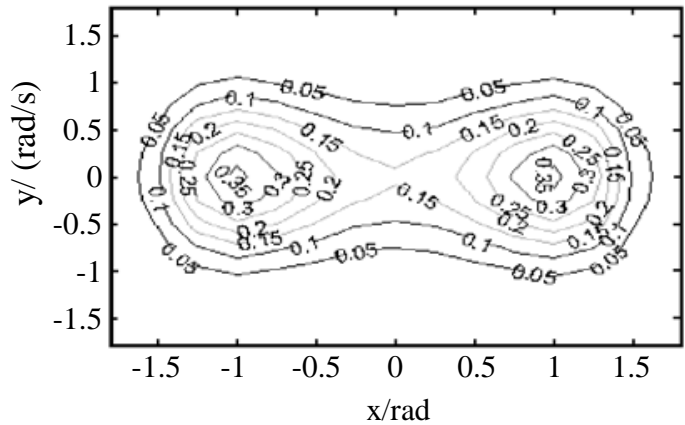

(c) $\mathrm{t}=15.15 / \mathrm{s}$

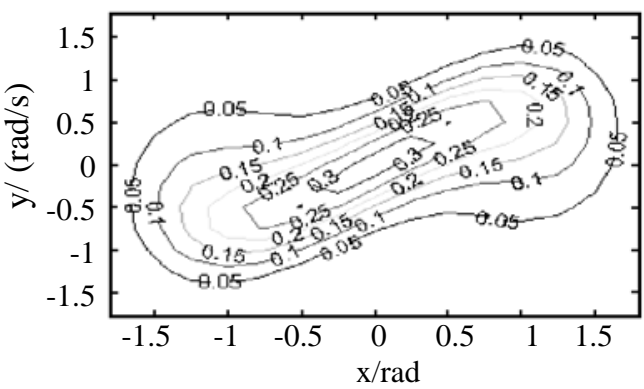

(b) $\mathrm{t}=9.85 / \mathrm{s}$

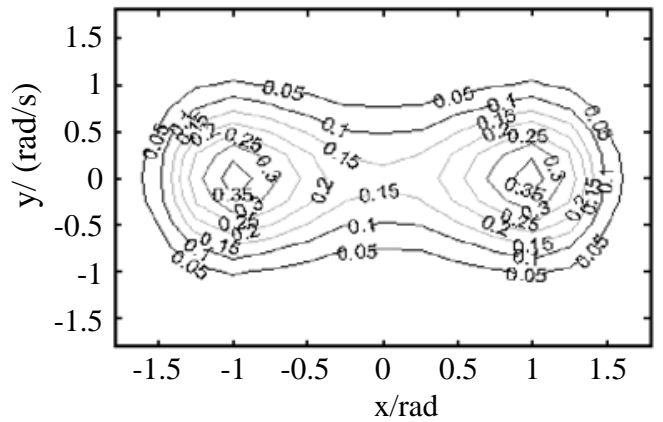

(d) $\mathrm{t}=21.21 / \mathrm{s}$

Fig. 2. Contour plots of the joint probability density function with $\left(d_{1}, k_{3}, D\right)=(0.185,1.0,0.087)$.

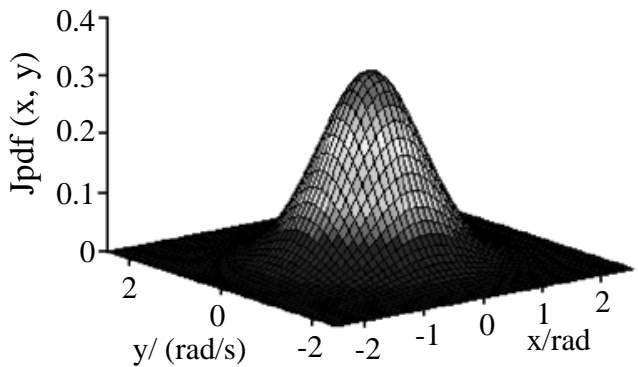

(a) $\mathrm{t}=0 / \mathrm{s}$

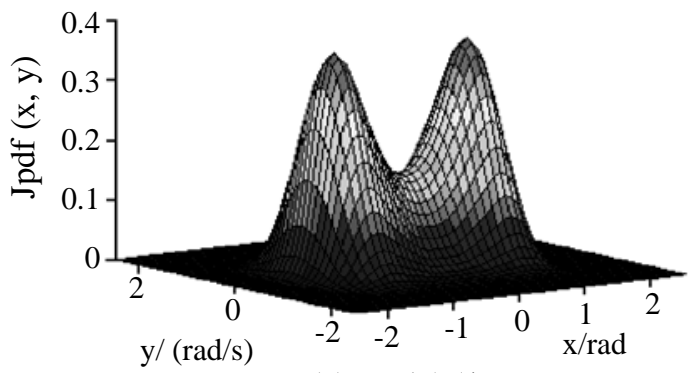

(c) $\mathrm{t}=15.5 / \mathrm{s}$

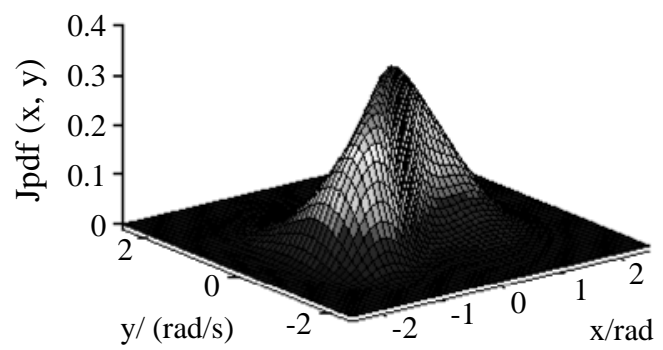

(b) $\mathrm{t}=9.85 / \mathrm{s}$

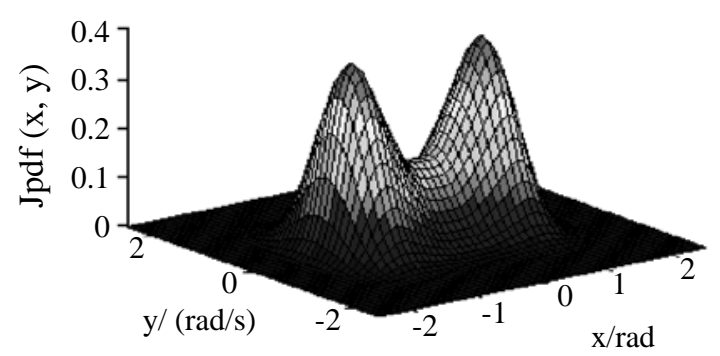

(d) $\mathrm{t}=21.21 / \mathrm{s}$

Fig. 3. Evolution of the joint probability density function with $\left(d_{1}, k_{3}, D\right)=(0.185,1.0,0.087)$.

threshold intensity of the white noise for onset of chaos analytically. The joint probability density function of ships' roll angle and the roll angular velocity is calculated by solving the Itô stochastic differential equation via the path integral method in the chaotic region. The stochastic chaotic response characteristic is further investigated via the probability density function and the numerical results of the system. It is found that the ships 


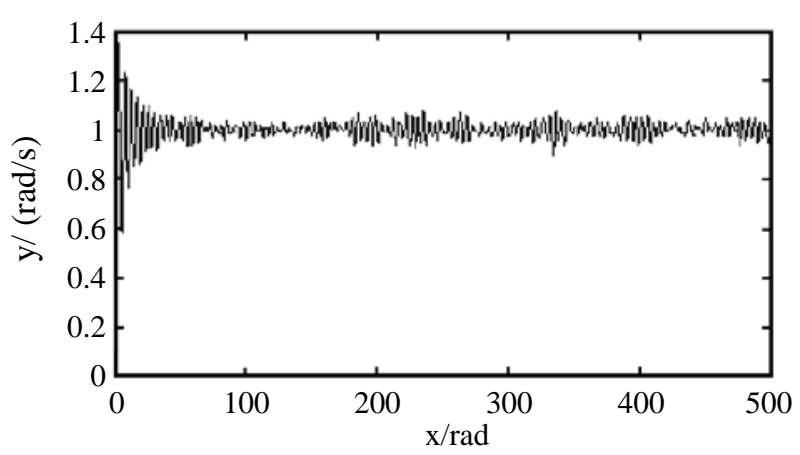

(a) Time history

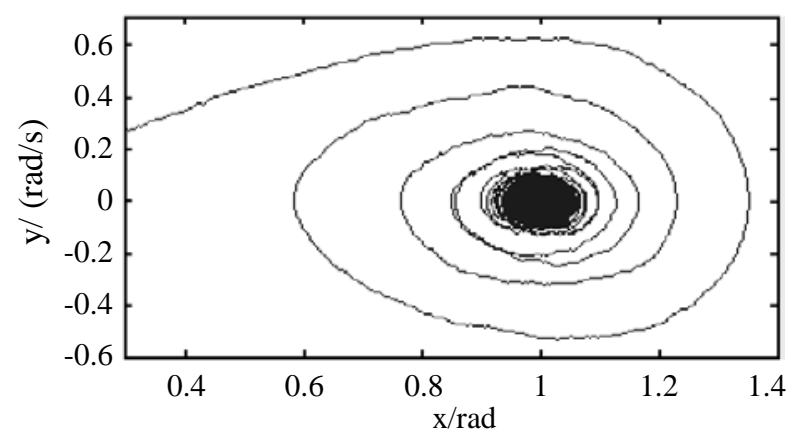

(b) Phase portrait

Fig. 4. Rolling response with $\left(d_{1}, k_{3}, D\right)=(0.185,1.0,0.05)$.

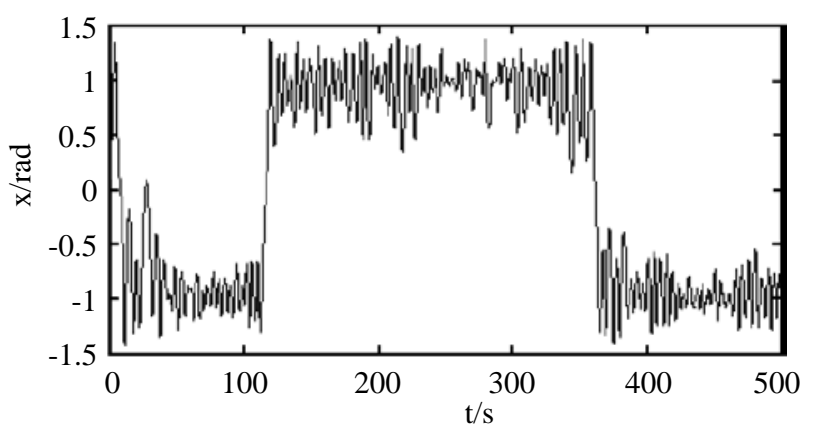

(a) Time history

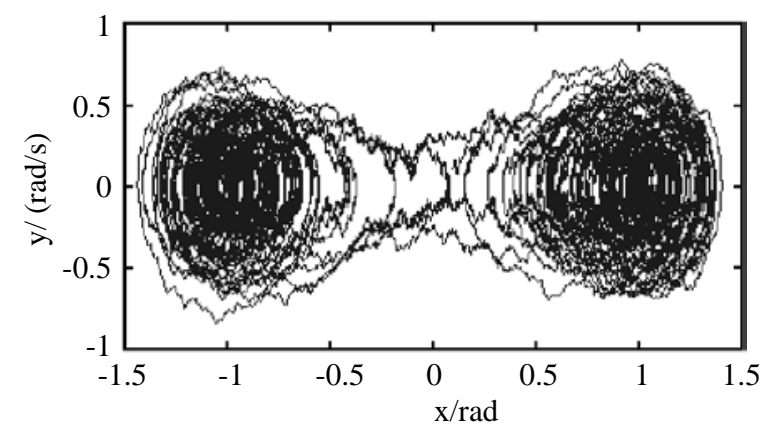

(b) Phase portrait

Fig. 5. Rolling response with $\left(d_{1}, k_{3}, D\right)=(0.185,1.0,2.3)$.

undergo stochastic chaotic motion when the real intensity of the white noise exceeds the threshold intensity and the stable probability density function has two peaks. The response of the system has two high probability states and it may jump from one state to another for high intensity of the white noise excitation. This is the unstable roll motion of ships and that will lead ships to capsizing. In real ship capsizing evaluation, the realistic wave such as the PM spectrum should be adopted. The realistic sea spectrum is produced by means of linear filter acting on white noise. Then four dimensions Ito differential equations should be solved.

\section{ACKNOWLEDGEMENT}

The authors are grateful to the National Natural Science Foundation of China for supporting this work (Grant No. 50279026).

\section{REFERENCES}

1. Falzarano, J.M., "Predicting Complicated Dynamics Leading to Vessel Capsizing," Ph.D. Dissertation,
Department of Naval Architecture, University of Michigan, Ann Arbor, MI (1990).

2. Francescutto, A., "Stochastic Modeling of Nonlinear Motions in the Presence of Narrow band Excitation," Proceeding of the Second International Offshore and Polar Engineering Conference, San Francisco, CA, pp. 91-96 (1992).

3. Hsieh, S.R., Troesch, A.W., and Shaw, S.W., "A Nonlinear Probabilistic Method for Predicting Vessel Capsizing in Random beam Seas," Proceedings of the Royal Society of London series A, Vol. 446, No. 1926, pp. 195-211 (1994).

4. Huan, L. and Yim, S.C.S., "Chaotic Roll Motion and Capsize of Ships under Periodic Excitation with Random Noise," Applied Ocean Research, Vol. 17, pp. 185-204 (1995).

5. Liu, Z.H. and Zhu, W.Q., "Homoclinic Bifurcation and Chaos in Simple Pendulum under Bounded Noise Excitation," Chaos Solutions \& Fractals, Vol. 20, pp. 593-607 (2004).

6. Naess, A. and Moe, V., "New Techniques for Path Integral Solution of the Random Vibration of Nonlinear Oscillators," Proceeding of $7^{\text {th }}$ International Conference of Structural Safety and Reliability, Balkema, 
Netherlands, pp. 795-801 (1998).

7. Naess, A. and Moe, V., "Efficient Path Integration Methods for Nonlinear Dynamic Systems," Probabilistic Engineering Mechanics, Vol. 15, pp. 221-231 (2000).

8. Sanchez, N.E. and Nayfeh, A.H., "Nonlinear Rolling Motion of Ships in Longitudinal Waves," International Shipbuilding Progress, Vol. 37, pp. 247-272 (1990).

9. Tang, Y.G., Jiang, D.N., and Zheng, H.Y., "Study on the Sub-harmonic Response of Ships Rolling and Pitching Coupling Motions," Journal of Tianjin University, Vol. 36, No. 2, pp. 183-186 (2003).
10. Wehner, M.F. and Wolfer, W.G., "Numerical Evaluation of Path-Integral Solutions to Fokker-Planck Equations," Physical Reviews A, Vol. 27, No. 5, pp. 2663-2670 (1983).

11. Yim, S.C.S. and Huan, L., "Unified Analysis of Complex Nonlinear Motions via Densities," Nonlinear Dynamics, Vol. 24, pp. 103-127 (2001).

12. Zhu, W.Q., Nonlinear Stochastic Dynamics and Control of Hamilton Theory System, Science Print, Beijing, P.R.C., pp.335-341 (2003). 